\title{
Acquisition and Dithering with the TMT IRIS On-Instrument Wavefront Sensor System
}

\author{
David R. Andersen*a ${ }^{*}$, Jennifer Dunn ${ }^{\mathrm{a}}$, James Larkin ${ }^{\mathrm{b}}$, Shelley Wright ${ }^{\mathrm{c}}$, Eric Chisholm ${ }^{\mathrm{d}}$, Jenny \\ Atwood $^{\mathrm{a}}$, Edward Chapin ${ }^{\mathrm{a}}$, Tim Hardy ${ }^{\mathrm{a}}$, Roger Smith ${ }^{\mathrm{e}}$, Gelys Trancho ${ }^{\mathrm{d}}$, \\ ${ }^{a}$ NRC Herzberg Astronomy \& Astrophysics, 5071 W. Saanich Rd, Victoria, BC, Canada; ${ }^{b}$ Dept. of \\ Physics and Astronomy, University of California, Los Angeles, CA 90095, USA; ${ }^{\circ}$ Dept. of Physics, \\ University of California, San Diego, 9500 Gilman Drive, La Jolla, CA 92093, USA; ${ }^{\mathrm{d}}$ Thirty Meter \\ Telescope Observatory Corporation, Pasadena, California 90807, USA; ${ }^{\mathrm{e}}$ Division of Physics, \\ Mathematics, and Astronomy, California Institute of Technology, Pasadena, CA 91125, USA
}

\begin{abstract}
IRIS is a first-light facility instrument for the TMT that operates as a client of the NFIRAOS MCAO system. IRIS is a collaboration between TMT, Caltech, the University of California, NAOJ and NRC Herzberg. IRIS contains three OnInstrument WaveFront Sensors (OIWFS) probes which together with On-Detector Guide Windows (ODGW) on the IRIS imager, pick off light from natural guide stars over a two arcminute diameter field of regard. Here, we present typical use cases for the OIWFS and ODGW including acquisition, dithering, and tracking non-sidereal targets while highlighting design choices that allow these operations to be performed in the minimal amount of time while achieving the required performance. We conclude with some potential changes that will be explored early in the final design phase.
\end{abstract}

Keywords: AO; tip/tilt/focus sensing; acquisition

\section{INTRODUCTION}

The Infrared Imaging Spectrograph (IRIS) is a cryogenic instrument under development for first-light operation of the Thirty Meter Telescope (TMT) [1]. IRIS is a client instrument of NFIRAOS, which is the first light, Multi-Conjugate Adaptive Optics (MCAO) system for TMT [2]. The IRIS science cryostat houses a "wide-field" imager and an integral field spectrograph (IFS) with a $0.84 \mu \mathrm{m}$ to $2.4 \mu \mathrm{m}$ wavelength range. The IRIS imager uses four Hawaii-4RG-10 detectors which yield a total field-of-view (FoV) of 34 x 34 arcsec at a platescale of 4 milliarcseconds (mas). The IFS offers four spaxel scales ranging from 4 mas to 50 mas, and is capable of generating up to 14,000 simultaneous spectra within a filled rectangular pattern. Three on-instrument wavefront sensors (OIWFS) are mounted in a separate enclosure atop the science cryostat [3]. These patrol the exterior perimeter of the two arc minute diameter field delivered by NFIRAOS and provide measurements of the tip/tilt, focus (TTF) and plate scale modes invisible to NFIRAOS and its laser guide star (LGS) wavefront sensors (WFS). IRIS mates to NFIRAOS through a support structure which in turn interfaces to a rotator ring. The science cryostat and OIWFS enclosure connect to this rotator, which enables IRIS to provide its own field de-rotation. The final component of IRIS is the services cable wrap, which routes the cables from the inside the OIWFS and science cryostat to the Nasmyth platform below the instrument and out to either the IRIS electronics cabinet or the TMT facility supplied services. A rendering of IRIS placed underneath NFIRAOS within the observatory is shown in Figure 1. The IRIS instrument on its own with the various subsystems labeled is shown in Figure 2. The IRIS instrument successfully completed its Preliminary Design Phase (PDP) in September 2017 and will initiate its Final Design Phase (FDP) in early 2018.

In these proceedings, we will focus on the OIWFS. This subsystem is critical for sensing tip/tilt, focus and platescale modes to which the LGS MCAO system is blind. These OIWFS operate in the near infrared (NIR) to take advantage of image sharpening provided by NFIRAOS. In this way, the OIWFS can make use of quite faint stars $(\mathrm{J}<22)$ and achieve a sky coverage of $\sim 50 \%$ even at the Galactic pole. In section 2, we will present aspects of the design of the OIWFS, touching on the optical, mechanical and software design. In section 3, we walk through some of the major OIWFS use cases dealing with acquisition, dithering and non-sidereal tracking. Finally in section 4, we develop some ideas for changes to the OIWFS design that will be explored early in the FDP.

*david.andersen@nrc-cnrc.gc.ca; phone 1250 363-8708

Updated 3/20/14 


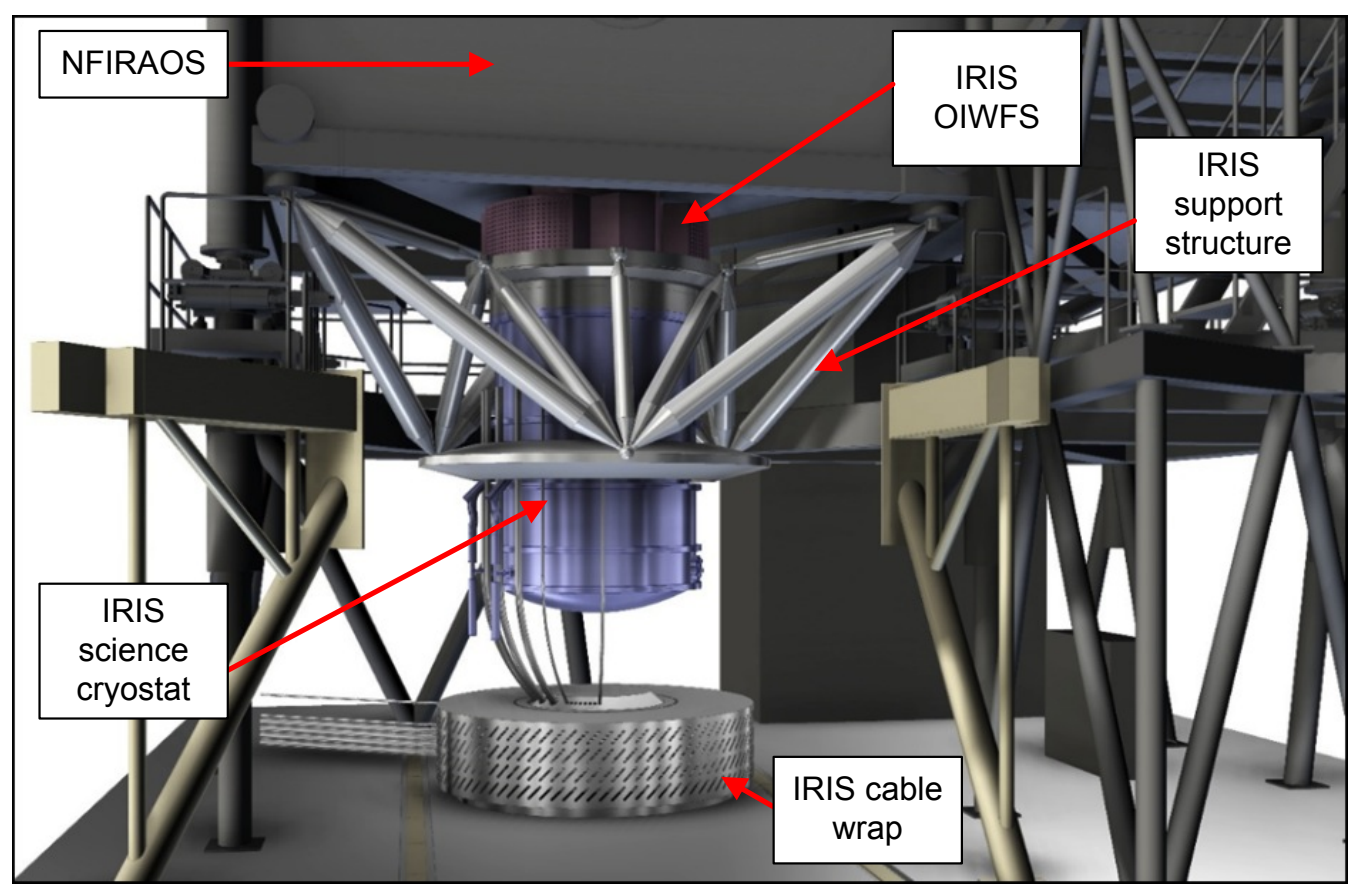

Figure 1. The IRIS instrument shown in-situ mounted to the up-looking port of NFIRAOS. The silver cylinder in the lower portion of the image is the IRIS cable services wrap, which is designed to prevent damage to IRIS cables as the cryostat rotates during operations. For scale, the blue dewar is 1.9 meters in diameter

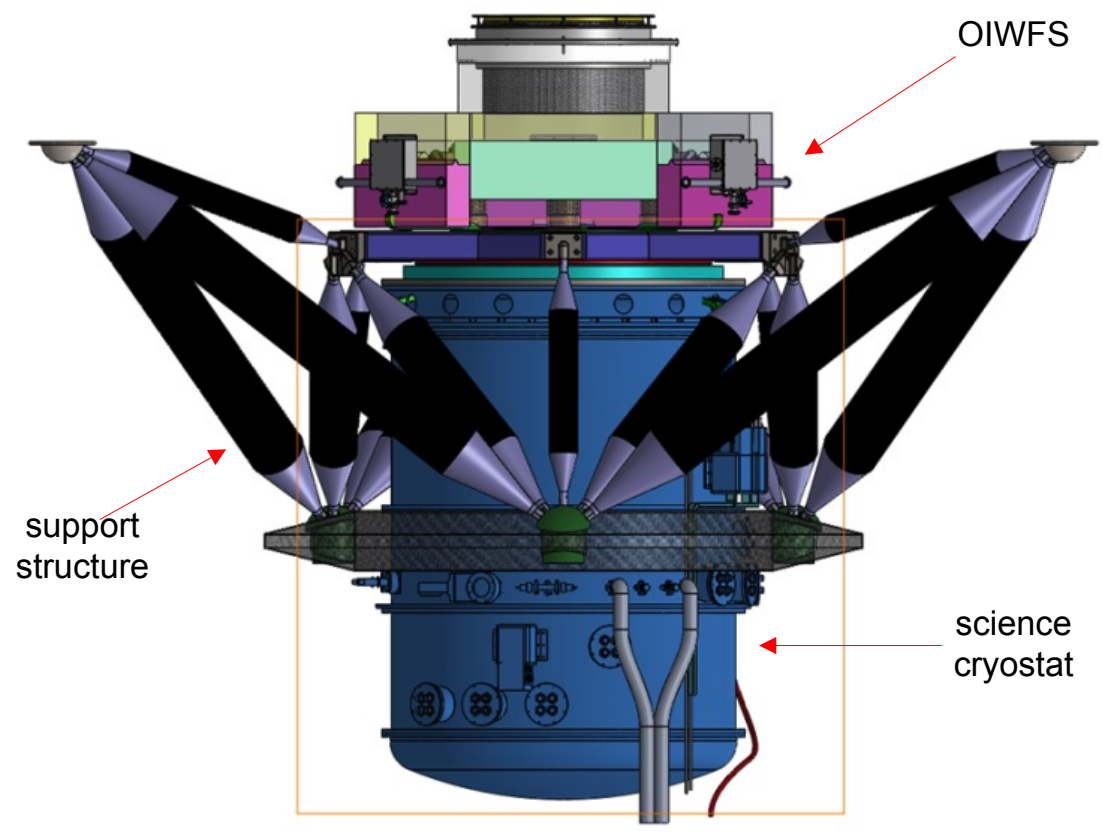

Figure 2. Close-up view of an external rendering of IRIS including the OIWFS, support structure and science cryostat 


\section{OIWFS DESIGN}

IRIS contains three OIWFS probe arms. These arms can be configured to sense tip/tilt (TT) or tip/tilt/focus (TTF). They operate in the NIR (1.1 to 2.3 microns). The wavelength range is chosen to cover all of $\mathrm{J}+\mathrm{H}+\mathrm{Ks}$ bands which maximizes the signal-to-noise of the natural guide star (NGS) detection while cutting off at 2.3 microns to block out most of the thermal infrared light. The plate scale was set to produce 6 mas/pixel (12 mas/pix in TTF mode) which critically samples a diffraction-limited NGS core. The OIWFS FOV has a goal to be five arcseconds to ease acquisition, but a smaller FOV can be acceptable.

\subsection{Optomechanical Design}

The OIWFS optical design is built around R-theta probe arms (Figure 3). A converter lens and fold mirror are close to the NFIRAOS delivered focal plane. A fixed field stop prevents scattered light from outside the FOV from reaching the detector. A collimator is mounted on a small linear stage to account for the focus errors introduced as the probe arms sweep across the curved NFIRAOS focal plane. Two trombone mirrors and two fold mirrors act as a periscope that preserves the path length as the probe stage moves radially. The periscope is on the axis of rotation of the theta stage. Elements after the $1^{\text {st }}$ fold mirror are fixed.

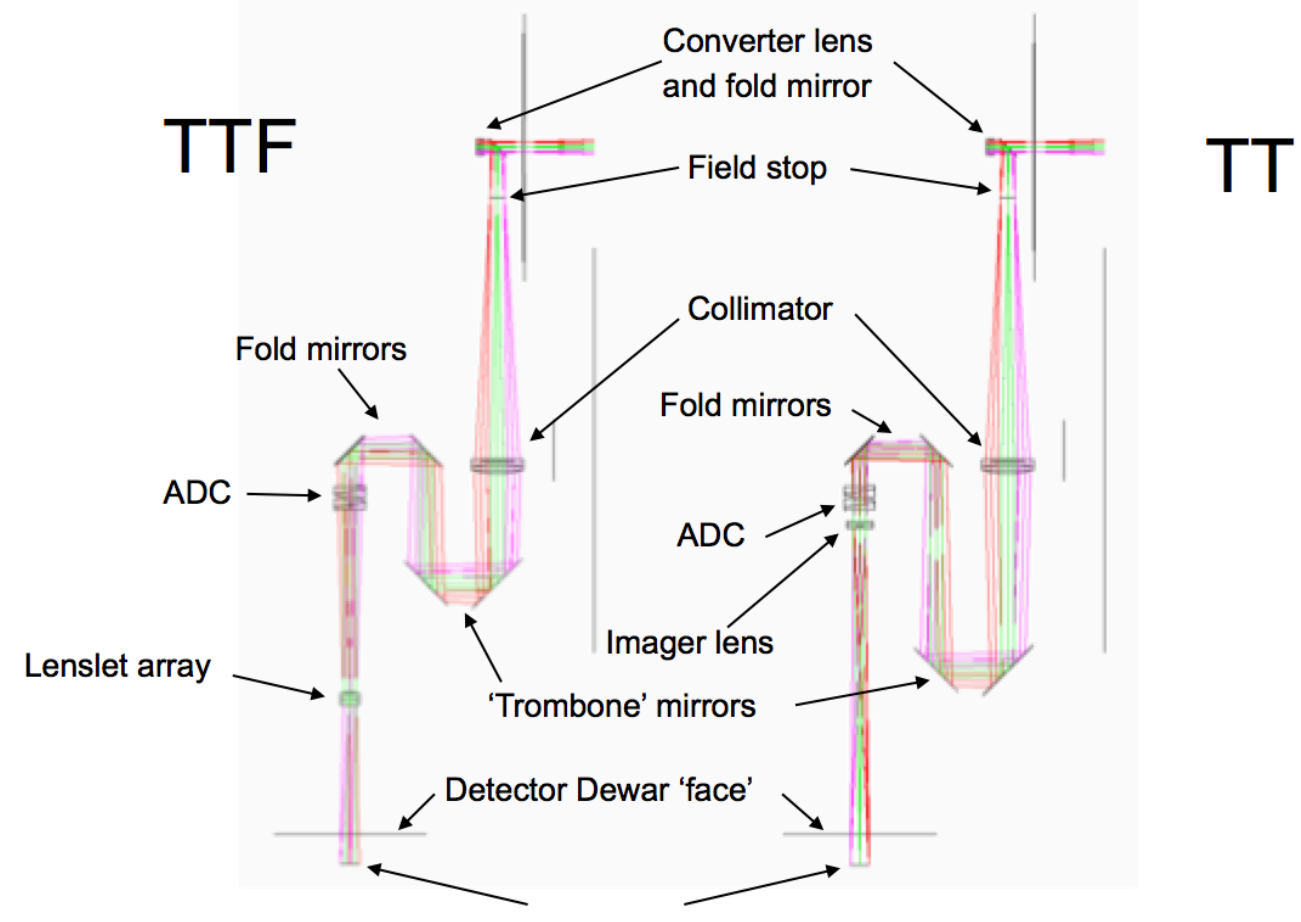

Figure 3: Optical design of tip/tilt and tip/tilt/focus versions of the OIWFS

The elements on the fixed portion of the arm include the atmospheric dispersion corrector (ADC) and an imager lens/lenslet array changer mechanism. When the imager lens is deployed, the OIWFS will be used to image a single star onto the detector (TT mode). When the lenslet array is deployed, four images of the star will be imaged onto the OIWFS detector (TTF mode).

The vignetting shadow from the probe is important because the probe may have to pick off NGS close to or in the imager FOV. The probe arm itself is very narrow and is close to the focal plane and will therefore lead to minimal vignetting if the probe extends across the science field. At its tip is a somewhat larger assembly. The $20.8 \mathrm{~mm}$ converter lens is held in a $25 \mathrm{~mm}$ diameter mount and sits $45 \mathrm{~mm}$ upstream of the NFIRAOS focal plane (Figure 5). Below the field lens, a pick-off mirror directs light to the collimator and then through the rest of the OIWFS optics. As shown, this probe tip can cause significant vignetting of the science field as it is physically larger and is further from the focal plane than the probe arm. For reference, the plate scale at this focal plane is $2.2 \mathrm{~mm} / \mathrm{arcsec}$. 

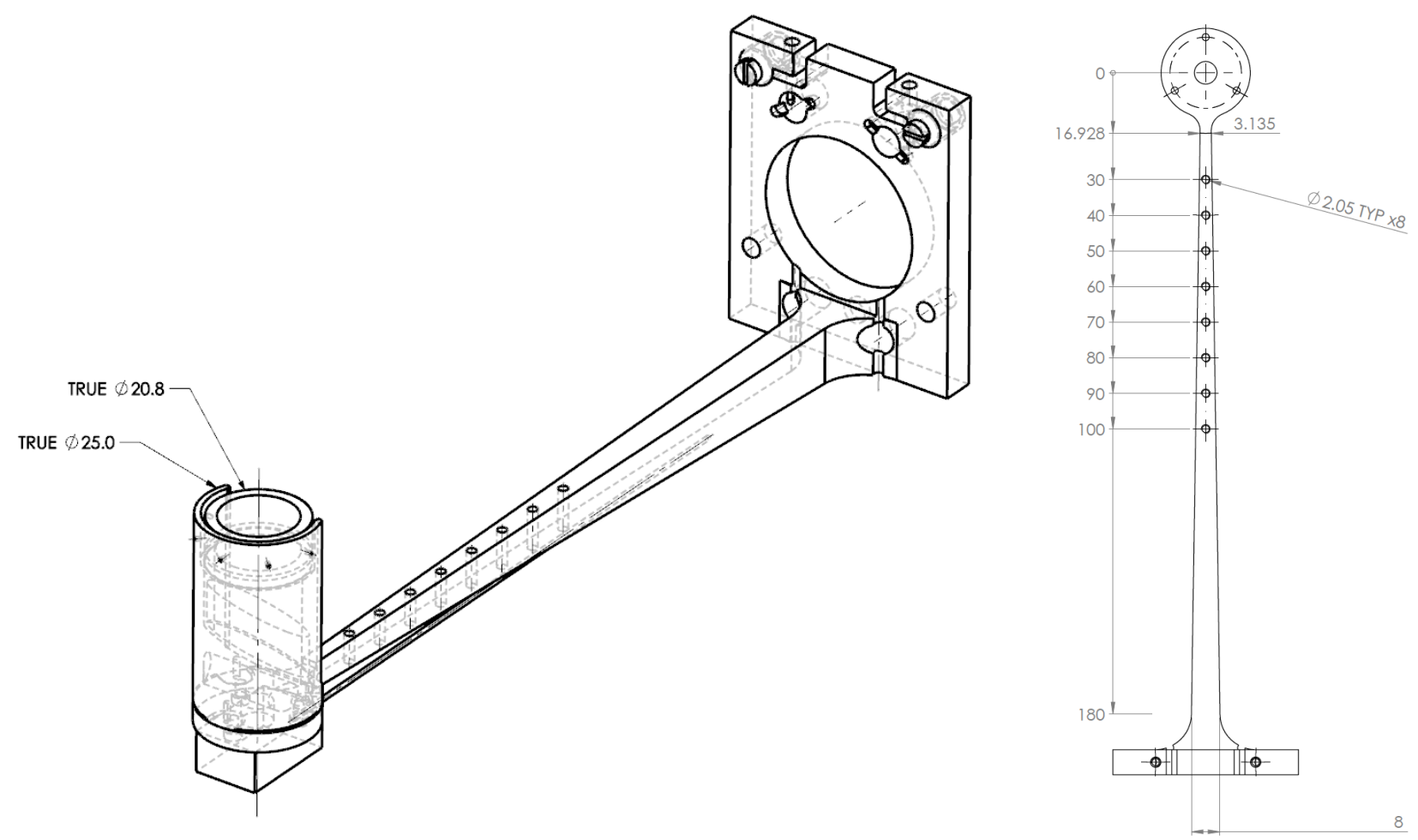

Figure 4: Mechanical design of OIWFS probe tip. The probe arm is very narrow and is situated close to the focal plane to minimize vignetting.
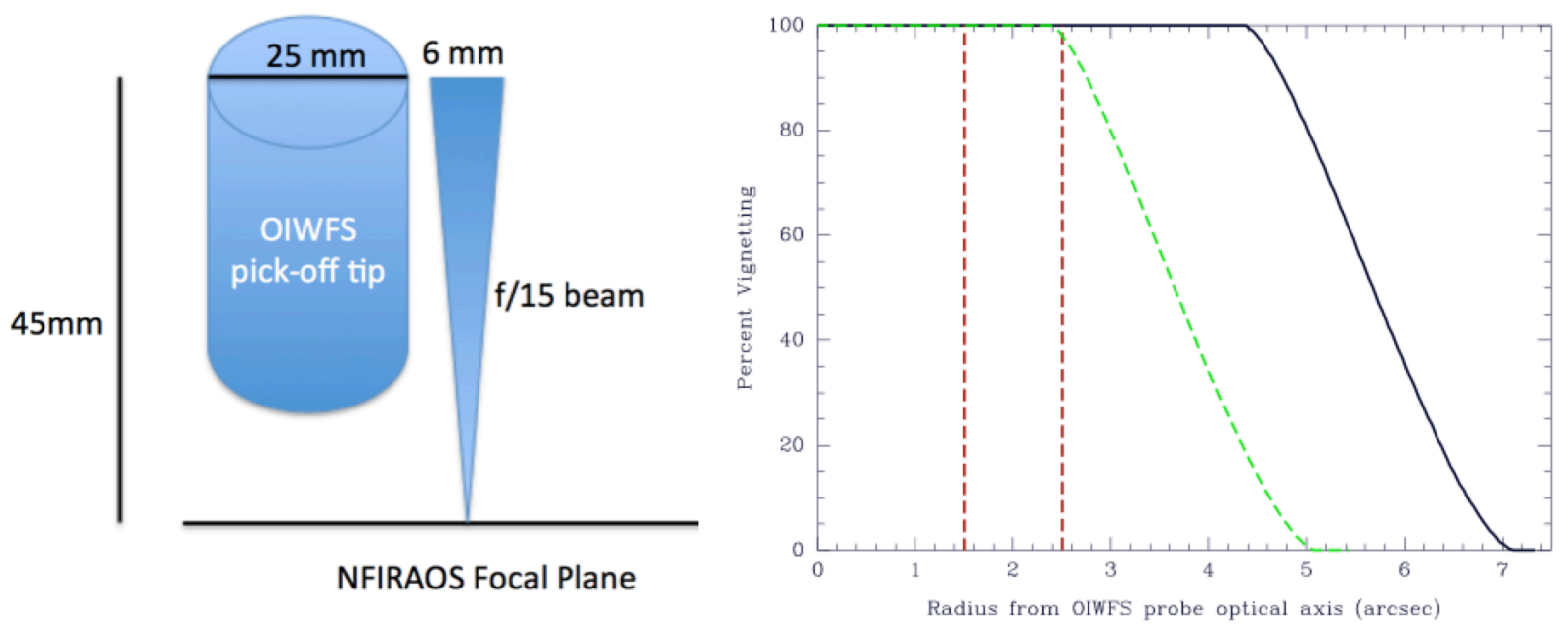

Figure 5: Left Panel: Geometry of the probe tip illustrating the vignetting in the current design. Right Panel: Vignetting as a function of radius from the center of the pick-off mirror for a 5 arcsec FOV baseline design (solid black line) and 3 arcsec FOV design (green dashed line).

The OIWFS mechanical design has been built to meet a demanding combination of requirements on speed, precision and range of motion (Figure 6). The three probe arms must be able to span the 2 arcminute field of regard ( $270 \mathrm{~mm})$. A requirement was set that each probe must be able to reach the center of the field (135 $\mathrm{mm}$ of travel), and must do so with 
a precision of 2 mas ( 4.5 microns). This latter requirement is in place to ensure that IRIS can be used to perform precision absolute astrometry (if the nearest reference star is within the NFIRAOS field of regard and is used as a OIWFS NGS, then the positions of objects falling on the IRIS imager can be determined relative to the OIWFS to high precision) and to ensure that observations of the same field taken at different epochs will have very nearly the same platescale. Finally, TMT is committed to observing efficiency. There are many top level requirements on how quickly science fields have to be acquired and how fast dithering patterns can be carried out. As discussed in the OIWFS use cases below, the OIWFS probe arms have to move up to $30 \mathrm{arcsec}$ in five seconds. Given the profile of the moves, this translates into a maximum probe speed of $12 \mathrm{arcsec} / \mathrm{sec}$. Given these demanding requirements, NRC has prototyped the OIWFS probe arm assembly and demonstrated that it is compliant even when operated at $-30 \mathrm{C}$ (the temperature inside NFIRAOS and the OIWFS enclosure).

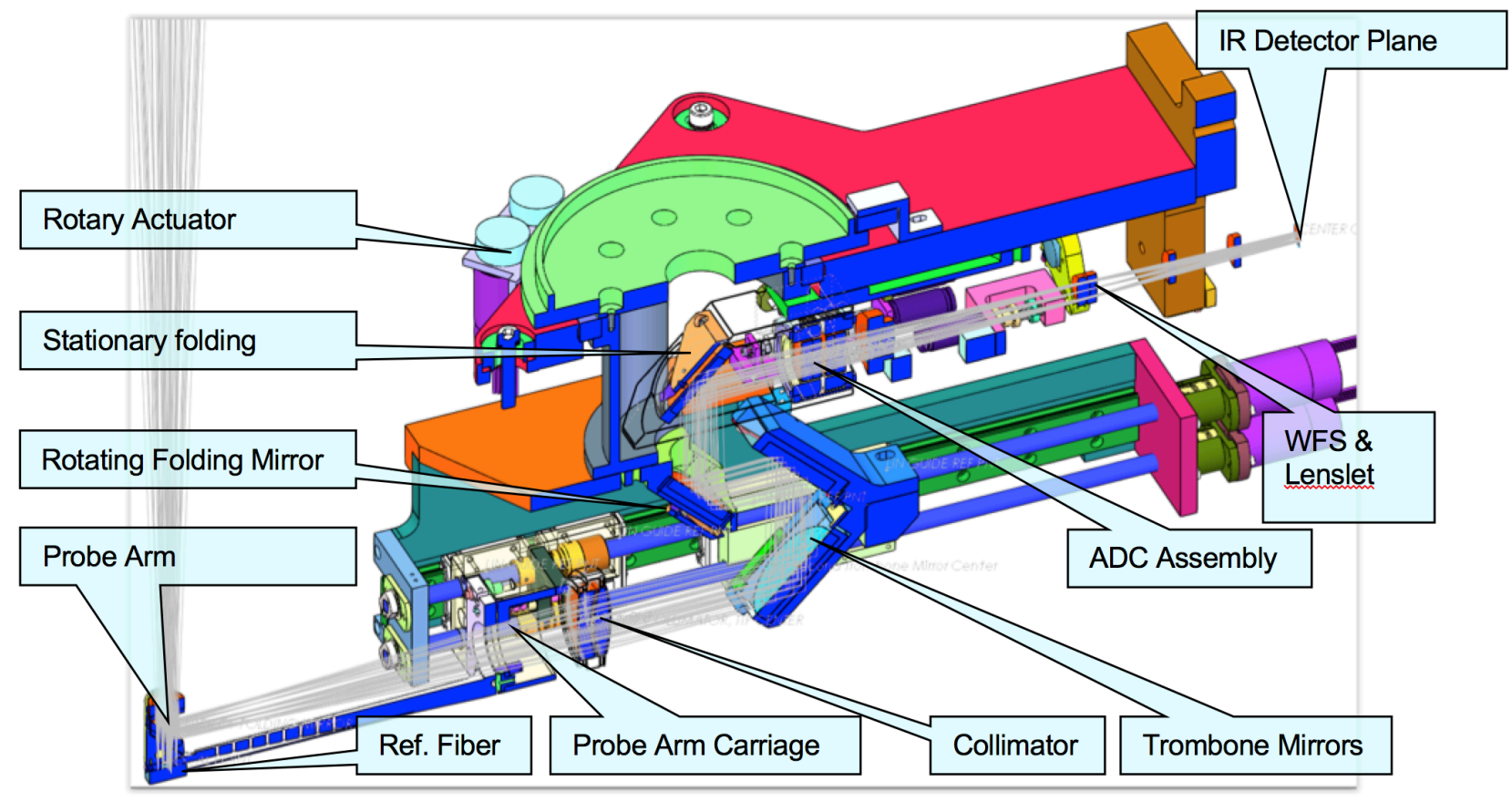

Figure 6: Illustration of OIWFS probe arm assembly (not including detector dewar).

\subsection{Software Design}

The TMT software design uses a hierarchical organization to control everything from the telescope to the instruments. The current vision has the executive software sending commands to all the secondary software systems. In turn, the OIWFS controller receives commands from two of these secondary subsystems, the AO Sequencer and the Telescope Control System (TCS; Figure 7). When a probe is commanded to move to a new location, the TCS provides the information that converts from on-sky coordinates to locations/positions in physical units (ADC rotation angles, pick-off arm locations and collimator set points). The AO Sequencer, meanwhile, sends commands to the detector controller. It specifies the mode of operation, the readout rate, and window location and size. Only the transfer of calibrated pixels does not obey this hierarchical scheme; the pixels are transferred straight from the IRIS detector controller to the NFIRAOS Real Time Controller (RTC).

The user will assign OIWFS to NGS using tiers when planning observations. The brightest star will typically be used as a fast TTF source. This "Tier 1" source is critical for NFIRAOS observations and cannot be switched without restarting the loop. The two Tier 2 stars are used to sense fast TT. Either an On-Detector Guide Window ${ }^{1}$ (ODGW) or OIWFS can also be read out slowly and used to measure any slow flexure (Tier 3). As an aside, the NFIRAOS pyramid WFS can be used in any of these roles or as a slow truth WFS.

\footnotetext{
${ }^{1}$ The ODGW is a subarray read out after each science row is read out from the Teledyne H4RG which enables a measurement of TT from the science detector. The ODGW will likely provide a Tier 3 slow TT measurement because it cannot produce a measurement as fast with as little readnoise as the OIWFS. There can be one ODGW per imager chip (4 total).
} 


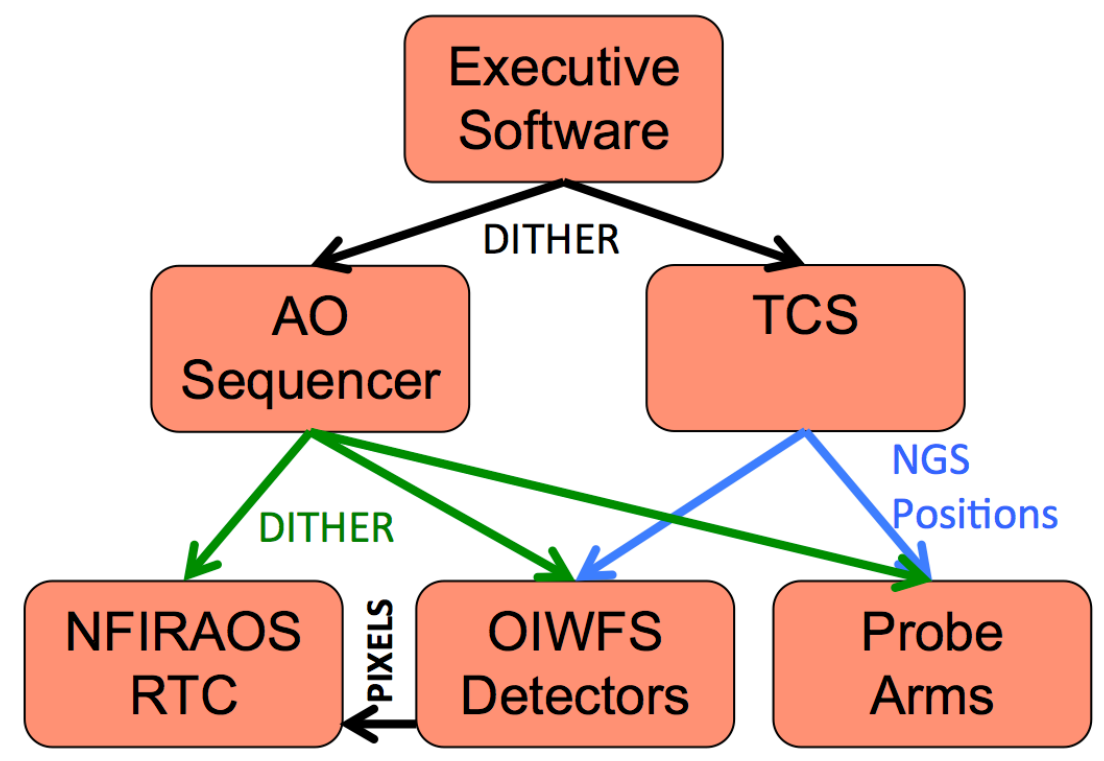

Figure 7: Overview of the SW control hierarchy of TMT and the IRIS OIWFS.

Each of the OIWFS includes a H1RG NIR array in the baseline design. Based on measurements made for the IRIS Conceptual Design Phase, the H1RG can achieve a read-noise of less than 3 electrons if a small region ( $4 \mathrm{x} 4$ pixels) is read out at $100 \mathrm{~Hz}$ or slower. The model for the operation for the OIWFS is similar to that of TRICK on Keck [4]. The detector controller can change the subwindow size and location to adapt to the different use cases, as described below. The basic scheme is shown in (Figure 8). For acquisition, a large region of interest (ROI) will be used. As the NGS is "captured" by the RTC, the ROI will shrink and the frame rate will increase until the window decreases to $4 \times 4$ pixels and is running at rates up to $800 \mathrm{~Hz}$ (although note that at $800 \mathrm{~Hz}$, the readnoise will be significantly higher than 3 electrons and will only be used with bright, photon-limited, NGS).

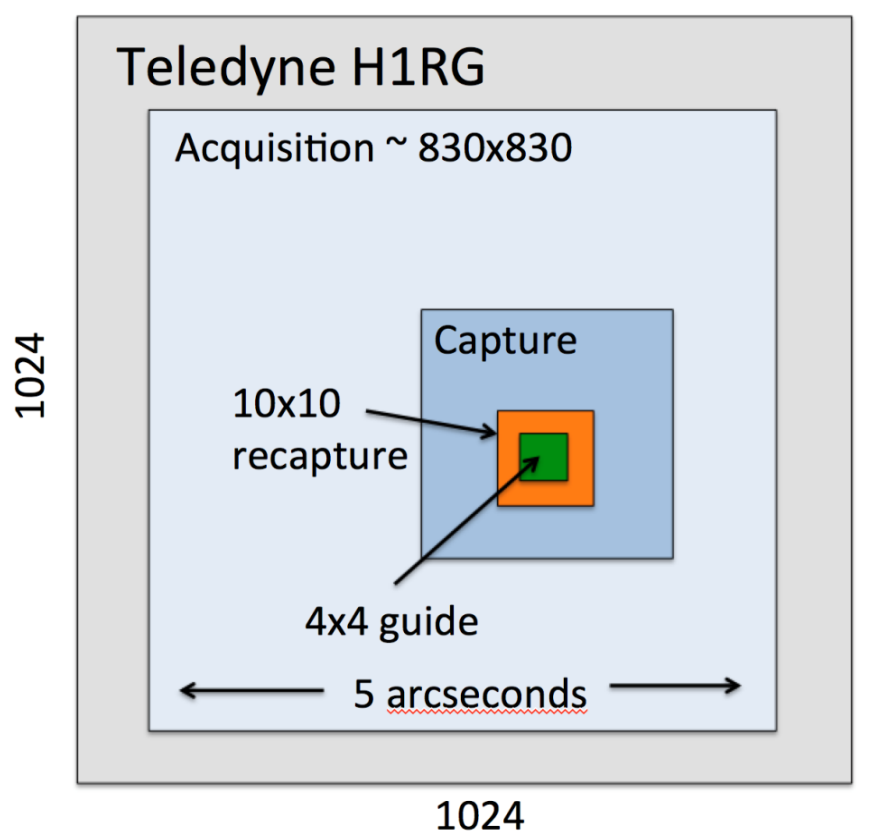

Figure 8: Illustration of capture and guide ROIs to be used with the OIWFS H1RG detectors. 


\section{OIWFS USE CASES}

\subsection{Acquisition}

One of the driving requirements for the OIWFS is that TMT requires that the overhead between observations be less than 3 minutes (if no instrument change is required). Most of this time is taken by the telescope as it slews from one spot on sky to another. Once the telescope is on target, there is limited time to acquire the field and start the observation. This requirement precludes the use of the OIWFS as the detector on which acquisition takes place. As shown in Table 1, the NGS would be captured $95 \%$ of the time if the telescope meets its ambitious 1 arcsec RMS blind pointing accuracy and the 5 arcsecond circular OIWFS FOV. That means that if the OIWFS were used to acquire the field, $5 \%$ of the time there would be no star observed and a blind spiral search would be needed which would cost valuable time. In reality, it may be difficult for TMT to reach this blind pointing accuracy in the early years of observations, and the probability of pointing errors significantly larger than 2.5 arcsec will be significantly higher than predicted from the tail of a Gaussian. Designing an OIWFS with a larger FOV is impractical as concerns over science field vignetting would be exacerbated.

Table 1: Acquisition success assuming 1 arcsec RMS error

\begin{tabular}{|l|l|l|}
\hline $\begin{array}{l}\text { WFS FoV } \\
\text { diameter }(\operatorname{arcsec})\end{array}$ & $\begin{array}{l}\text { Probability of blind } \\
\text { acquisition success }\end{array}$ & $\begin{array}{l}\text { Required Detector size } \\
\text { (assuming 6 mas/pix) }\end{array}$ \\
\hline 6.0 & 0.99 & $1000^{2}$ array \\
\hline 5.0 & 0.95 & $833^{2}$ array \\
\hline 4.0 & 0.86 & $667^{2}$ array \\
\hline 3.0 & 0.67 & $500^{2}$ array \\
\hline
\end{tabular}

Therefore, we plan to acquire fields using either the IRIS science detector (16.4 arcsec FOV for each quadrant) or the NSEN acquisition camera (20 arcsec FOV, but with a coarser 30 mas pixel scale on a commercial InGaAs SWIR detector). The acquisition sequence only has time to identify the brightest NGS in the field. TMT will be pointed to center the brightest NGS on either IRIS or the NSEN acquisition camera. The LGS will be launched and the high order NFIRAOS loops will be closed to concentrate the NGS light for acquisition. In the meantime, the OIWFS will all be commanded to their expected final position relative to the science target using a collision avoidance algorithm [5]. Once the star has been detected and the pointing plus catalog error measured, the telescope will offset to the science target coordinates which will bring 3 NGS onto their respective pre-positioned OIWFS probes.

The Tier 1 (fast TTF) star will be acquired first with the OIWFS (usually the brightest NGS just used for acquisition). Telescope pointing accuracy over offsets less than 1 arcminute should be very good and the star will almost assuredly be picked up by the OIWFS. The detector will use a pre-defined table of window regions of interest (ROI) to zoom in onto the star. The initial ROI will be $\sim 50 \times 50$ pixels in the $2 \times 2$ Shack-Hartmann mode $(0.6 \times 0.6$ arcsec $)$ and then if the star is detected, the window will shrink and the star will be driven by the AO system to the expected position on the detector (nominally the center of each subaperture; Figure 8 illustrates the process for a TT star). If during any step in this process, the star is not in this window, the window will enlarge in steps (following the pre-defined table) until the full detector is read out. In normal operations with the Teledyne H1RG detector baseline, the ROI will eventually shrink to $4 \mathrm{x} 4$ pixels (four $4 \mathrm{x} 4$ pixel subregions for a TTF) to reduce readnoise as described above.

As long as the other NGS have relative catalog errors less than $0.5 \operatorname{arcsec}$ RMS with respect to the Tier 1 star, the other 2 probes should be able to pick their NGS with a probability of $>99 \%$ (even, it should be noted, if the FOV of the OIWFS were reduced to 3 arcsec in the interests of alleviating the vignetting issue). In the unlikely event that one of these Tier 2 NGS is not detected, the OIWFS would move through a spiral search pattern (e.g. for a OIWF probe with a 3 arcsec FOV, Figure 9 shows four positions in red that would sweep out most of a 6 " diameter area). The four moves to complete this pattern could be executed in $\sim 0.4$ seconds. Reading out the $1 \mathrm{k}^{2}$ ROI with sufficient sampling to sense even faint NGS will take roughly 1 second for the H1RG. Assuming $\sim 2 \mathrm{sec}$ were spent at the first pointing (because the observer can not know if the object is found until after the $1^{\text {st }}$ exposure) and $1 \mathrm{sec}$ exposures were taken at the other 4 positions, the first round of the spiral search could be complete within $\sim 7.6$ seconds. 
If a NGS is still not detected, the reason will most likely be that the catalog photometric or astrometric errors are large or that the guide target is extended. In these cases, the observer will have to select an alternative NGS if possible, and reacquire the NGS for the field. As long as the Tier 1 star (fast TTF) is successfully acquired, however, performance may be sufficient to perform a given science case even if one of the other NGS turns out not to be a valid fast TT target.

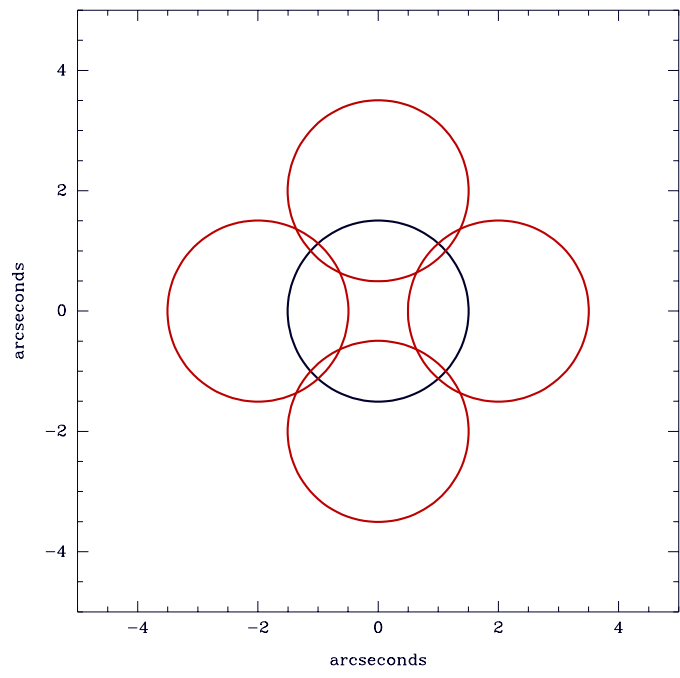

Figure 9: Example of spiral search pattern (red) that could be used by 3 arcsec OIWFS probe.

\subsection{Dithering}

One of the most common use cases involving the OIWFS probes is dithering. Dithering for IRIS is defined as moves small enough that the NGS targets can be tracked by the OIWFS during a small telescope move. This limits the size of a dither to $\sim 45$ arcsec. TMT requirements again prioritize the minimization of overhead (Table 2). To achieve this, the AO loops need to be kept closed during the dither (re-acquisition starting with a large ROI on the OIWFS would be too time consuming). We have therefore defined two types of allowable dithers: 1) Probe dithering, in which the probe assemblies move in coordinated motion with the telescope to follow the star, and 2) On-chip dithering, in which detector ROIs move in coordinated motion with the telescope while the probe assembly is static.

Table 2: TMT dithering overhead requirements

\begin{tabular}{l|l}
\hline Offset & Allowed Overhead \\
\hline 1 arcsec & Less than 2 seconds \\
\hline 5 arcsec & Less than 2.5 seconds \\
\hline $10 \operatorname{arcsec}$ & Less than 4 seconds \\
\hline 30 arcsec & Less than 5 seconds \\
\hline
\end{tabular}

During any dither, the window will increase in size (to roughly 0.3 arcsec depending on how well the OIWFS and telescope can be coordinated). The speed of the readout will have to be reduced during this time and the gain reduced in the RTC. The probe or the center of the window will move in coordination with the telescope. At the end of the move, the window will shrink and the gain increased with minimal lost time. This process will have to involve a careful coordination between the AO sequencer which controls the RTC and the OIWFS detector controllers and the TCS which moves the telescope and provides a stream of NGS positions to the OIWFS components controller.

On-chip dithering will be useful when small precision offsets are done. Since the probe arm assembly won't move during these offsets, the NGS move can be controlled accurately within $\sim 10 \%$ of a OIWFS pixel. A larger FOV for the OIWFS allows for larger on-chip dithers, but given that the OIWFS probe arm mechanisms have an accuracy of 2 mas RMS (30\% of 
a OIWFS pixel) and that ODGW can potentially be used to control large moves (each science detector quadrant is 16.4 arcseconds on a side), the value of having a large OIWFS FOV for on-chip dithering is diminished.

During probe dithers, it may be possible that the dither moves one of the NGS out of range of a probe arm. As long as the Tier 1 fast TTF star remains locked, however, the dither can be successfully completed. The OIWFS that lost its NGS can be used to acquire a new Tier 2 fast TT star. Depending on the expected AO performance without the TT star, science observations can resume while a new TT star is acquired.

\subsection{Non-Sidereal Guiding}

The TMT requires that science instruments can observe non-sidereal targets that are moving as fast as $1.5 \mathrm{arscsec} / \mathrm{sec}$ relative to the sidereal tracking rates. If the OIWFS were forced to track NGS that moved across the field at this rate, the NGS would pass out of the capture range in less than 40 seconds. However, objects moving this fast are near earth objects (NEOs) that will also be bright enough that they can serve as their own AO guide star on the NFIRAOS PWFS. This alleviates the requirement that the OIWFS has to allow non-sidereal observations when sources are moving this fast, but luckily, most non-sidereal targets move much slower.

Objects further from the Earth (planets, moons, asteroids, and comets) will have a non-sidereal tracking rate slower than one arcsec per minute. We have prepared use cases involving tracking background stars with the OIWFS while observing solar system targets. For objects moving 1 arcsec per minute, guide probes will reach their limits typically every 10 to 15 minutes. If all OIWFS are set as fast TTF probes, observations can continue with only minimal impact on science while a probe "crab walks" from one NGS to another. These crab walks by probes have been modeled using the same anti-collision algorithms [5], and we believe that a hand-off can be made in $\sim 10$ seconds.

\section{POTENTIAL FUTURE CHANGES TO THE OIWFS DESIGN}

We have developed a optomechanical, electrical and software preliminary design of the OIWFS subsystem of IRIS that meets the requirements of the TMT observatory. As part of this design work, we have examined possible changes to the OIWFS optomechanical assembly and the OIWFS detector that may further improve the performance of this system.

As shown in Figure 5, vignetting of the probe arm assembly could impact IRIS science if the NGS are close to the central $34 \times 34$ square arcsec IRIS imager FOV. We can mitigate this issue through reducing the OIWFS FOV and by removing the field lens in front of the pick-off mirror. Of these two potential changes, removing the field lens and adding power to the pick-off mirror would have a much bigger impact than just shrinking the FOV alone. In the final design phase of IRIS, we will spend some effort to explore whether this change can be made.

A possibly even more impactful change is being considered as we explore whether to switch detectors from a Teledyne H1RG to a Leonardo NIR APD [6]. The NIR APD has all the properties one desires of a WFS detector: low read noise, high achievable frame rate, deeper full well and a large ROI. The lower read noise would have a significant impact on sky coverage. The noise equivalent angle for a faint target that is readnoise limited with a H1RG would shrink significantly with the APD. The readouts electronics for the NIR APD will be capable of reading out the entire array at rates faster than $800 \mathrm{~Hz}$ in which case the relative complexity of coordinating the detector ROI and probe movements during acquisition and dithering would be greatly reduced or even eliminated. The deeper full well reduces the risk of saturation and persistence on the OIWFS detectors and allows users to use even brighter NGS as guide stars if they are available. The one downside of making this change of detectors is the risk associated with H1RG detectors being a proven technology while NIR APDs are still new and relatively unproven in the field. Given the timeline of the IRIS project, we will explore whether it is possible to upgrade to a 512x512 Leonardo APD during the final design phase. This format of NIR APDs should be available during the IRIS FDP, and while a $512 \times 512$ pixel device limits the FOV to be 3 arcsec or less, the downsides to shrinking the FOV seem to be acceptable in light of the other advantages. The acquisition process already baselines using a different detector to acquire the brightest target so a 3 arcsec FOV will be sufficient. The smaller FOV even has an advantage in that the magnitude of science field vignetting will be reduced.

During the final design phase of IRIS, these changes will be considered by the IRIS team and our colleagues at TMT, and the design of the IRIS OIWFS will be advanced to a state ready for fabrication. The critical software interfaces to the AO sequencer and the TCS will also be refined and clarified during this phase so that TMT + NFIRAOS + IRIS will work as an efficient system on-sky. 


\section{REFERENCES}

[1] Larkin, J.E., et al., “The Infrared Imaging Spectrograph (IRIS) for TMT: instrument overview,” Proc. SPIE 9908, id. 99081W (2016).

[2] Herriot, G., et al., "NFIRAOS: first facility AO system for the Thirty Meter Telescope," Proc. SPIE 9148, id. 914810 (2014).

[3] Dunn, J., et al. "The Infrared Imaging Spectrograph (IRIS) for TMT: multi-tiered wavefront measurements and novel mechanical design," Proc. SPIE, 9908, id. 9908A9 (2016).

[4] Castellá, F. et al. "Status and new developments with the Keck I near-infrared tip-tilt sensor," Proc. SPIE, 9908, id. 990925 (2016).

[5] Chapin, E., et al. "Infrared Imaging Spectrograph (IRIS) for TMT: motion planning with collision avoidance for the on-instrument wavefront sensors," Proc. SPIE, 9913, id. 99130T (2016).

[6] Hall, D., Baker, I., Finger, G. "Towards the next generation of L-APD MOVPE HgCdTe arrays: beyond the SAPHIRA 320 x 256,” Proc. SPIE, Volume 9915, id. 991500 (2016). 